\title{
Effects of Housing Conditions on the Development of Wet Skin Lesions in the NOA Mouse
}

\author{
Taizo KONDO, Toshio KONDO, Yasuhisa SHIOMOTO, and Akira MOMII
}

Division of Pharmacology, Drug Safety and Metabolism, Otsuka Pharmaceutical Factory, Inc., 115 Tateiwa, Muya-cho, Naruto, Tokushima 772-8601, Japan

\begin{abstract}
The effects of housing on the onset time and prevalence of wet skin lesions were investigated in NOA mice, which spontaneously develop these lesions at a high rate. Wet skin lesions developed earliest in mice that were housed individually. For mice that were housed in groups, the lesions developed earlier in mice with non-littermate group housing than in mice with littermate group housing. The prevalence of lesions was in the following order: individual housing > non-littermate group housing > littermate group housing. These results suggest that socio-psychological factors are involved in the etiology of wet skin lesions in the NOA mouse. Under individual housing conditions, two other novel characters of the NOA mouse were also observed, specifically, development of dry skin and wet skin lesions at the tail root. These characteristics developed early and with high prevalence and were easily observed on external examination. Therefore, these novel characteristics observed in NOA mice are potential markers of the psychological state of the animals.
\end{abstract}

Key words: animal model, dry skin, individual housing, NOA mouse, psychosocial stress

\section{Introduction}

The Naruto Research Institute Otsuka Atrichia (NOA) mouse is a hair-deficient mutant [11] that has been established as an inbred strain in our facility. This mouse strain spontaneously develops ulcerative and erosive wet skin lesions (Fig. 1) at a high rate [11]. Since it is thought that mast cells and $\operatorname{IgE~[11]~as~well~as~}$ eosinocytes [12] may be involved in the development and aggravation of these skin lesions, the NOA mouse is expected to be useful as an animal model of allergic dermatopathy.

Based on the findings obtained from detailed exami- nations and strain-maintenance, the development of wet skin lesions in the NOA mouse appears to differ among various housing conditions. It is well known that differences in housing density and environmental conditions can affect the behavior, physiological functions, and immune function of rodents due to psychological stress [1, 3-6, 9, 15, 17, 19]. In humans, the involvement of psychological factors and stress in atopic dermatitis (AD), a representative allergic dermatopathy, is widely recognized $[2,14,16]$. For these reasons, psychological stress might play a role in the development and aggravation of wet skin lesions in the NOA mouse. It is expected that confirmation of this

(Received 26 October 2004 / Accepted 5 January 2005)

Address corresponding: T. Kondo, Division of Pharmacology, Drug Safety and Metabolism, Otsuka Pharmaceutical Factory, Inc., 115 Tateiwa, Muya-cho, Naruto, Tokushima 772-8601, Japan 


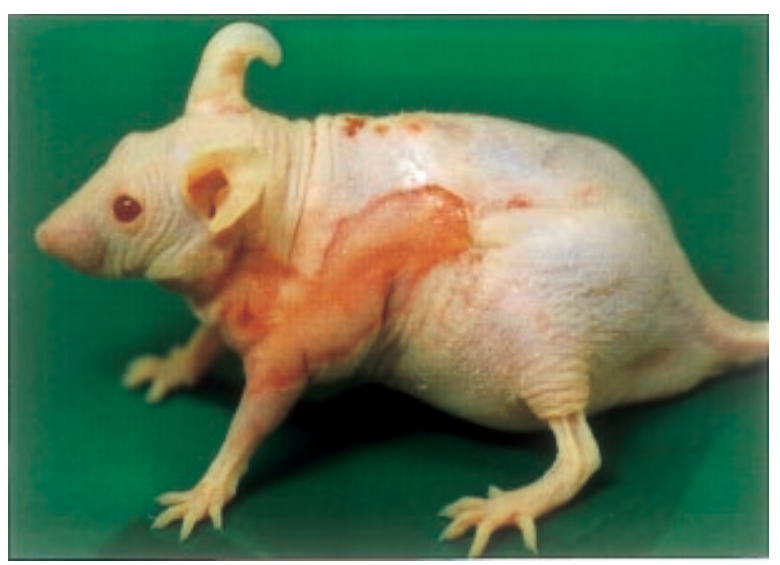

Fig. 1. Wet skin lesions in an NOA mouse (male, 25 weeks of age).

would lead to the acceptance of the NOA mouse as a useful animal model for analyzing the relationships between dermatopathy and psychological factors.

In the present study, the effects of different housing conditions on the onset time and prevalence of wet skin lesions in NOA mice were investigated.

\section{Materials and Methods}

This study was carried out using SPF animals under barrier-system environmental conditions. All of the NOA mice used were bred in our facility. The animals were maintained under the following environmental conditions: temperature $23 \pm 2^{\circ} \mathrm{C}$, relative humidity $55 \%$ $\pm 10 \%$, ventilation rate $13-16$ exchanges/h at an airflow speed of $25 \mathrm{~cm} / \mathrm{s}$ or less, and a 12-h light-dark cycle (lights on 7:00 A.M. to 7:00 P.M.). Plastic (polycarbonate $)$ animal cages $(170[\mathrm{~W}] \times 280[\mathrm{D}] \times 130[\mathrm{H}]$ mm, CLEA Japan, Inc., Tokyo, Japan) were used. For individual housing, the cages were partitioned at the center with an aluminum plate, which halved the living space and permitted housing of 2 animals per cage. ALPHA-dri ${ }^{\mathrm{TM}}$ (Shepherd Specialty Papers, Inc., Watertown, TN, USA) was used as bedding. The cages and bedding were replaced once every week. The animals were supplied with pellet food CRF-1 sterilized by $\gamma$-ray irradiation (Oriental Yeast, Co., Ltd., Tokyo, Japan) and filter-sterilized tap water ad libitum.

Twenty-two 4-week-old male NOA mice were the subjects of the study. After littermate group housing (2-3 mice/cage) for 1 week, the mice were allocated to three groups at 5 weeks of age: (1) individual housing, (2) non-littermate group housing, and (3) littermate group housing. Eight mice were allocated to the individual housing group, 9 mice to the non-littermate housing group ( 4 mice/cage and 5 mice/cage), and 5 mice to the littermate housing group ( 2 mice/cage and 3 mice/cage). The mice were visually examined once a week for 25 weeks up to the age of 30 weeks to assess their skin condition.

The present study was approved by the Committee on the Care and Use of Laboratory Animals of Otsuka Pharmaceutical Factory, Inc.

\section{Results}

The changes in the prevalence of wet skin lesions in NOA mice are shown in Fig. 2. Throughout the observation period, the development of wet skin lesions on the ventral side of the tail root (immediately above the anus) (Fig. 3A), a novel and characteristic change, was observed in the NOA mice, mainly those in the individual housing group. Figure $2 \mathrm{~A}$ shows the changes in the prevalence of wet skin lesions after exclusion of this type of lesion. Wet skin lesions developed earliest in mice that were housed individually. In mice that were housed in groups, the lesions developed earlier in mice with non-littermate housing than in mice with littermate housing. The prevalence of lesions was in the following order: individual housing $>$ non-littermate group housing $>$ littermate group housing.

In the individual housing group, many mice exhibited frequent and intense biting of the ventral side of the tail root shortly after the start of individual housing. Changes in the prevalence of wet skin lesions in the tail root region alone are shown in Fig. 2B. Onset occurred in the following order: the individual housing group, the non-littermate housing group, and the littermate housing group. Prevalence increased in the same order. These wet skin lesions in the tail root region were classified into three types: lesions that resolved within a few days, chronic lesions that gradually expanded toward the femoral or lower abdominal region, and recurrent lesions.

The development of dry skin, mainly in the cervicodorsal region, which is a new and characteristic change in NOA mice, was also observed in the individual housing group (Fig. 3B). The external appearance of an 

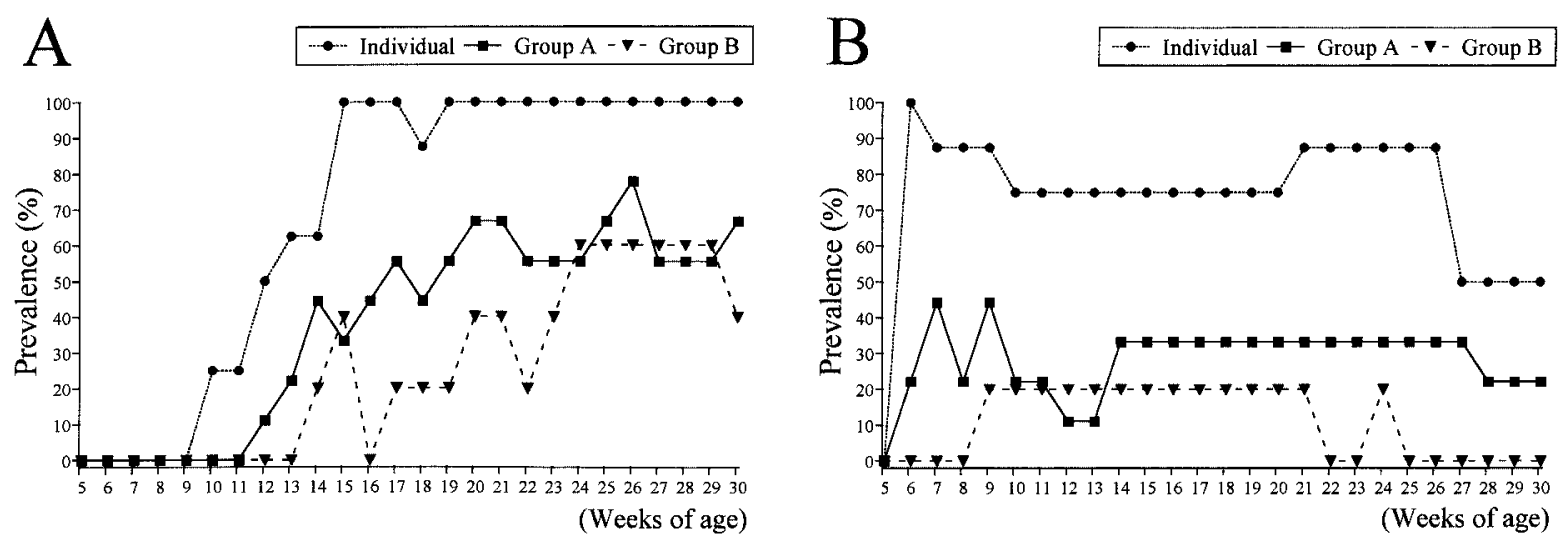

Fig. 2. Changes in the prevalence of wet skin lesions. A: Lesions other than those at the tail root. B: Lesions at the tail root alone. Individual: Individual housing at 5 weeks of age $(n=8)$. Group A: Non-littermate group housing $(n=9,2$ cages). Group B: Littermate group housing ( $\mathrm{n}=5,2$ cages).
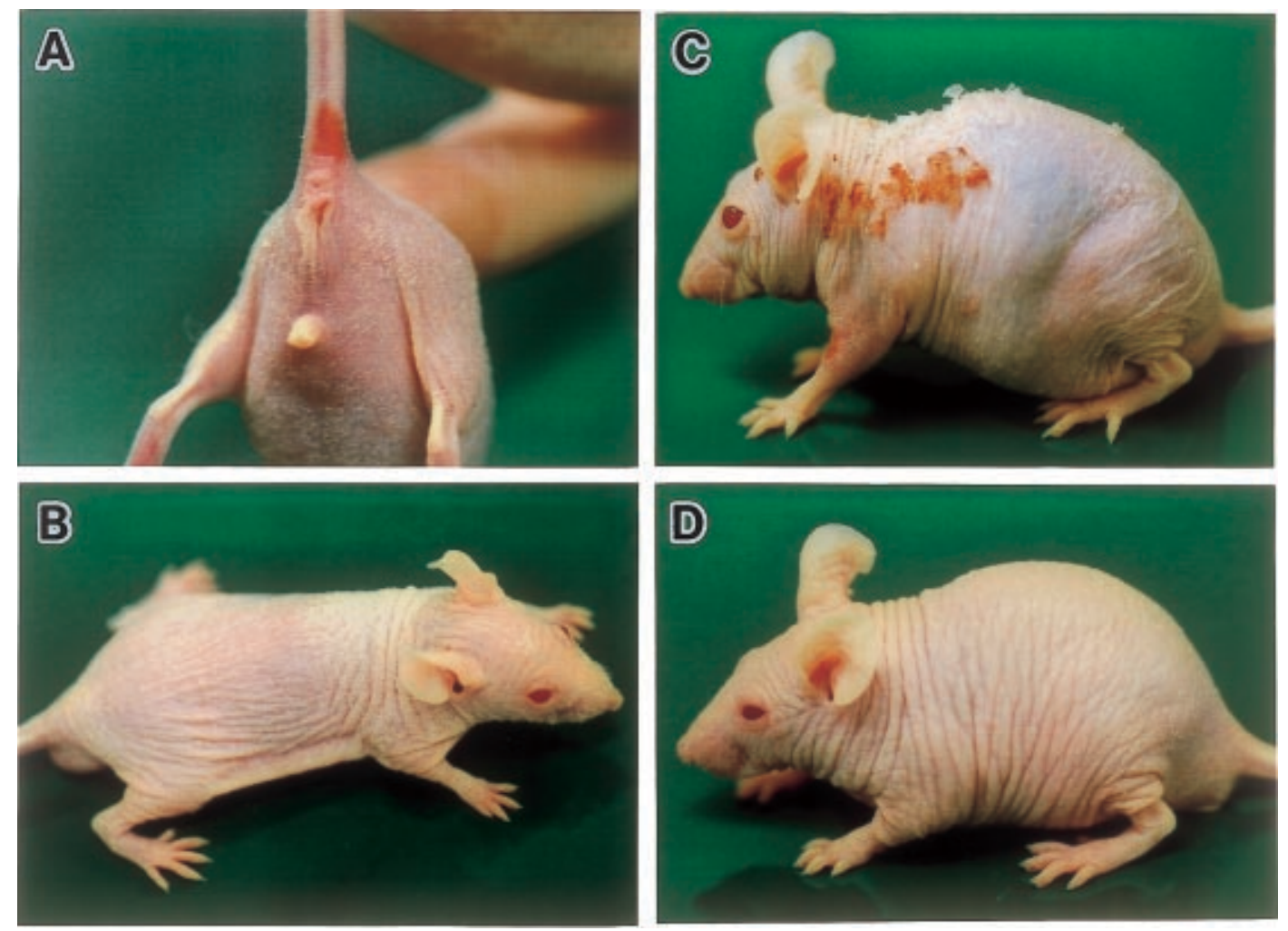

Fig. 3. External appearance of NOA mice (all males). A: Wet skin lesion at the tail root with individual housing (after 1 week of individual housing, 6 weeks of age). B: Dry skin with individual housing (after 3 weeks of individual housing, 8 weeks of age). C: Dry skin and wet skin lesions with individual housing (after 10 weeks of individual housing, 15 weeks of age). D: Normal skin (group housing, 10 weeks of age).

NOA mouse under group housing conditions is shown in Fig. 3D. Smooth skin is clearly seen over the entire body. In contrast, Fig. 3B shows a mouse with pityroid scales, mainly in the cervico-dorsal region, and dry skin over the entire body. All NOA mice developed this condition within 4 weeks of the start of individual hous- 
ing. When individual housing was continued, the scales became lamellar and wet skin lesions with crust formation developed in the dorsal region (Fig. 3C). In addition, frequent scratching of the dorsal region with the hind legs was observed in many of the mice.

External examination confirmed that the site of development of wet skin lesions in the NOA mice was not limited. It was also confirmed that in the head and cervico-dorsal regions, the changes were limited to wet skin lesions (mostly erosive) with crust formation, as shown in Fig. 3C. No severe ulcerative lesions developed, such as those extending from the upper foreleg to the lateral abdominal region shown in Fig. 1.

\section{Discussion}

The findings of the present study have confirmed that different housing conditions affect the onset time and prevalence of wet skin lesions in the NOA mouse. The severity of such skin lesions was ranked in the following order: individual housing $>$ non-littermate group housing $>$ littermate group housing (data not shown). Individual or isolated housing of rodents is widely employed as a model of psychological stress, particularly in the field of psycho-neurological medicine $[3,5,9,15,17]$. When group-rearing mice in cages, littermate group housing is considered to be a more stable social environment than non-littermate group housing $[4,5,17]$. Therefore, socio-psychological factors appear to be involved in the onset and aggravation of wet skin lesions in the NOA mouse.

The populations per cage differed for the littermate housing group versus the non-littermate housing group, due to the expected maximum number (2-3) of male littermate NOA mice. Therefore, it would be desirable to conduct further studies in equal populations for the both housing groups to confirm our findings.

In mice, stress in the rearing environment is known to affect skin morphology and function. Under individual housing conditions, Swiss white mice were found to show depilation and ulcerative skin lesions at the depilation site caused by scratching [3], BALB/c mice were found to show abnormal proliferation of epidermal cells [15], and hairless mice (HR-1) were found to show delayed recovery from artificial skin barrier disorders caused by tape stripping [9]. Under overcrowded housing conditions, hairless mice (HR-1) were found to exhibit epidermal thickening, accelerated epidermal proliferative activity, a decrease in corneocyte size, and delayed recovery from artificial skin barrier disorders caused by tape stripping [9]. BALB/c mice were found to show moderate exfoliation and slight wrinkle formation on the epidermis and also lowering of skin surface conductance [1].

It has long been known that stress affects the immune function in the mouse [19]. It has been reported that individual housing decreased immunity [5] and that short-term (2 days) isolated single housing suppressed the development of allergic contact dermatitis caused by a hapten, whereas long-term (30 days) isolated single housing exacerbated such dermatitis [15].

Based on the above findings, it may be inferred that various effects of stress are involved in the onset and aggravation of wet skin lesions in the NOA mouse.

In the external examinations conducted in the present study, the wet skin lesions of the head and cervicodorsal region of the NOA mice did not become severely ulcerated, but in many cases remained as erosive lesions with encrustation. Mice cannot reach their head or cervico-dorsal region with their mouths. In most cases, animals respond to itching or pain of the skin by biting and licking, but when this occurs in the head or cervico-dorsal region, which cannot be reached by the mouth, animals respond by scratching with their hind legs. NOA mice frequently bit the wet skin lesions in the lateral abdominal region and lumbar region and also bit lesions in the breast region by lifting the skin with their forelegs. We believe that biting and licking are involved in the development of severe ulcerative lesions in the NOA mouse. Severe ulcerative lesions develop due to the aggravation of mild wet skin lesions (erosive lesions) by the mechanical stimulation and tissue destruction associated with biting and licking, as well as the actions of saliva and contamination. Therefore, severe ulcerative lesions are thought to be a secondary disease state. The wet skin lesions that develop in the NOA mouse are much more severe than those observed in human atopic dermatitis; however, humans do not bite itching or painful skin lesions, and this difference appears to be reflected in the disease state. Therefore, the examination of lesions of the head and cervico-dorsal region, early-stage lesions, and the regions surrounding ulcerative lesions may be important in analyzing the usefulness of the NOA mouse as a 
possible animal model of allergic dermatopathy. The infiltration of mast cells [11] and eosinocytes (unpublished data), which are presumed to be involved in the development of such lesions, was confirmed in surrounding regions, and in particular, large numbers of these cells were observed around ulcerative lesions.

Two novel characteristics were observed in the NOA mice in this study: specifically, the formation of wet skin lesions in the tail root region and the development of dry skin, mainly in the cervico-dorsal region, under individual housing conditions.

The wet skin lesions at the tail root (Fig. 2A) developed earlier than the previously reported wet skin lesions [11] (Fig. 2B). Particularly under individual housing conditions, the onset was early and the prevalence was high. Although skin lesions at the tail root were also observed with group housing, the prevalence was low. In non-littermate group housing, the onset was earlier and the prevalence was higher than in littermate group housing. These results suggest that socio-psychological factors are involved in the development of wet skin lesions at the tail root. Since frequent and intense biting of the tail root was observed in many of the mice immediately after the start of rearing under individual housing conditions, the development of wet skin lesions at the tail root is presumably related to biting. In group housing, mice with wet skin lesions at the tail root also showed biting of that region. Kobayashi [10] demonstrated the presence of scratching behavior independent of itch stimulation in human AD patients, so-called "addictive scratching" or "scratch dependence", and discussed its possible involvement in the aggravation, recurrence, and chronicity of AD. The behavior of biting the tail root observed in NOA mice is similar to the behavior of AD patients.

The development of dry skin was observed in all of the NOA mice that were housed individually, but was not observed in any of the mice that were housed in groups. These results suggest that socio-psychological factors appear to be involved in the development of dry skin. Therefore, by exploiting the dry skin with individual housing, the NOA mouse strain could possibly serve as a useful animal model for evaluating the efficacy of topical moisturizers. No suitable animal model for evaluating the efficacy of topical moisturizers has been reported to date.

The involvement of stress in AD, a typical allergic dermatopathy in humans, has been recognized for many years $[2,14,16]$ and stress is considered to be one of the causes of $\mathrm{AD}[8,18]$. Lammintausta et al. [13] reported that psychic stress is the most common aggravating factor in adult $\mathrm{AD}$ and Cole et al. [7] reported that psychotherapy is effective for the treatment of $\mathrm{AD}$. Therefore, the NOA mouse can be considered as an animal model for analyzing the relationships between dermatopathy and psychological factors. The two novel characteristics observed in NOA mice, i.e., dry skin and wet skin lesions at the tail root developing under individual housing conditions, could be used as markers of the psychological state of the animals. These characteristics develop early and with high prevalence and can easily be observed on external examination.

The NOA mouse will be marketed by CLEA Japan, Inc., and has potential for widespread use. We expect that the NOA mouse will prove to be useful in the following areas: 1) as an animal model for investigating the relationships between skin conditions and psychological states; 2) as a marker of psychological state, with dry skin and wet skin lesions at the tail root prepared by individual housing; and 3 ) as a disease model for allergic dermatopathy, as has previously been proposed [11].

The abstract of this report was presented at the 50th Annual Meeting of the Japanese Association for Laboratory Animal Science (2003, Saitama, Japan).

\section{References}

1. Aioi, A., Okuda, M., Matsui, M., Tonogaito, H., and Hamada, K. 2001. Effect of high population density environment on skin barrier function in mice. J. Dermatol. Sci. 25: 189-197.

2. Allerhand, M.E., Gough, H.G., and Grais, M.L. 1950. Personality factors in neurodermatitis: a preliminary study. Psychosom. Med. 12: 386-390.

3. Banerjee, U. 1972. Somatic physiologic and behavioral effects of prolonged isolation in male mice and behavioral response to treatment. Physiol. Behav. 9: 63-67.

4. Bartolomucci, A., Palanza, P., Gaspani, L., Limiroli, E., Panerai, A.E., Ceresini, G., Poli, M.D., and Pannigiani, S. 2001. Social status in mice: behavioral, endocrine and immune changes are context dependent. Physiol. Behav. 73: 401-410.

5. Bartolomucci, A., Palanza, P., Sacerdote, P., Ceresini, G., Chirieleison, A., Panerai, A.E., and Parmigiani, S. 2003. Individual housing induces altered immuno-endocrine responses to psychological stress in male mice. Psychoneuroendocrinology 28: 540-558. 
6. Calham, J.B. 1962. Population density and social pathology. Sci. Amer. 206: 139-148.

7. Cole, W.C., Roth, H.L., and Sachs, L.B. 1988. Group psychotherapy as an aid in the medical treatment of eczema. J. Am. Acad. Dermatol. 18: 286-291.

8. Hanifin, J.M. 1984. Atopic dermatitis. J. Allergy Clin. Immunol. 73: 211-222.

9. Ishida, H., Mitsui, K., Nukaya, H., Matsumoto, K., and Tsuji K. 2003. Study of active substances involved in skin dysfunction induced by crowding stress. I. Effect of crowding and isolation on some physiological variables, skin function and skin blood perfusion in hairless mice. Biol. Pharm. Bull. 26: 170-181.

10. Kobayashi, M. 2000. Investigation of scratching behavior in atopic dermatitis patients. Jpn. J. Dermatol. 110: 275282 (in Japanese).

11. Kondo, T., Shiomoto, Y., Kondo, T., and Kubo, S. 1997. The NOA mouse: a new hair-deficient mutant: a possible animal model of allergic dermatitis. Mouse Genome 95: 698-700.

12. Kondo, T., Shiomoto, Y., Kubo, S., and Kyogoku, M. 1999. NOA mouse: A new strain of mouse with a possibility of animal model for allergic dermatitis. Exp. Anim. 48: 315.

13. Lammintausta, K., Kalimo, K., Raitala, R., and Forsten, Y. 1991. Prognosis of atopic dermatitis: a prospective study in early adulthood. Int. J. Dermatol. 30: 563-567.

14. McLaughlin, J.T., Shoemaker, R.J., and Guy, W.B. 1953. Personality factors in adult atopic eczema. Arch. Derm. Syph. 68: 506-516.

15. Nakano, Y. 1999. Stress worsens contact sensitivity. Clin. Immunol. 32: 365-371 (in Japanese).

16. Obermayer, M.E., Blair, R., Fiske, C., Levitt, N., Rush, S., Storkan, M.A., and Glenn, E. 1952. Correlation of emotional status and reactivity to cutaneous stimuli in functional dermatoses. Arch. Derm. Syph. 65: 291-302.

17. Palanza, P., Gioiosa, L., and Parmigiani, S. 2001. Social stress in mice: gender differences and effects of estrous cycle and social dominance. Physiol. Behav. 73: 411-420.

18. Rajika, G. 1986. Atopic dermatitis: correlation of environmental factors with frequency. Int. J. Dermatol. 25: 301-304.

19. Riley, V. 1975. Mouse mammary tumors: alteration of incidence as apparent function of stress. Science 189: 465467. 